\title{
Analysis of the Torque Characteristics of a Multi-Degrees of Freedom Surface Permanent-Magnet Motor
}

\author{
Dong-Woo Kang ${ }^{1}$, Sung-Chul Go ${ }^{1}$, Sung-Hong Won ${ }^{2}$, Seung-Bin $\mathrm{Lim}^{3}$, and Ju Lee ${ }^{1 *}$ \\ ${ }^{1}$ Department of Electric Engineering, Hanyang University, Seoul 133-070, Korea \\ ${ }^{2}$ Dongyang Technical College, Seoul 152-080, Korea \\ ${ }^{3}$ Korea Institute of Energy Technology Evaluation and Planning, Seoul 135-280, Korea
}

(Received 19 November 2009, Received in final form 17 December 2009, Accepted 29 December 2009)

\begin{abstract}
The multi-degrees of freedom surface permanent-magnet motor (Multi-D.O.F. SPM) has several degrees of freedom operations that are defined as the "roll", "yaw", and "pitch". Normally, the torque that is generated to rotate a rotor includes ripples. The analysis of the torque ripples is important for improving motor performance. In terms of the electric analysis, torque ripple occurs as a result of many factors, including the rotor and stator structures, the distribution of the air-gap flux density, and the waveform of the current in the coils. In particular, the torque ripple is an important factor in the stable operation of the Multi-D.O.F. SPM. Therefore, in this work, the torque ripple was analyzed using various types of magnetization for the permanent magnet. An improved model was proposed for the Multi-D.O.F. SPM based on this analysis.
\end{abstract}

Keywords : SPM, spherical motor, magnetization pattern, torque ripple

\section{Introduction}

The Multi-Degrees of Freedom Induction Spherical Motor was originally developed by E. R. Laithwaite of Manchester University in the 1950s [1]. Various types of Multi-D.O.F. motors have been proposed in order to operate a rotor with multi-degrees of freedom. In the 1980s, Vachtsevanos and Davay proposed a spherical type of multi-D.O.F. motor, and Kaneko developed a DC servo type of multi-D.O.F. motor. Rashid and Khalil researched a spherical type of stepping multi-D.O.F. motor $[2,3]$. Recently, a variable reluctance spherical motor was developed by Kok-Meng Lee. The analytical models of the torque generation and operation characteristics continue to be researched [4].

\section{Multi-D.O.F. SPM Structure}

The multi-D.O.F. SPM had the same structure as a normal surface permanent-magnet motor, and the permanent magnet was attached onto the rotor. The permanent magnet generated an electromagnetic field. However, in

*Corresponding author: Tel: +82-2-2220-0342

Fax: +82-2-2295-7111, e-mail: julee@hanyang.ac.kr
Fig. 1-(b), the multi-D.O.F. SPM had a spherical shaped rotor that differed from the normal cylindrical shaped rotor of the SPM. This structure was necessary in order to operate the rotor in multi-degrees of freedom in Fig. 2. Therefore, the structure of the spherical shaped rotor and stator maintained a constant air-gap distance during rotor tilt operation. The rotor had two operation modes, a tilt mode and a rotate mode, in Fig. 2. In the tilt mode, the rotor tilted the command position, which required the Alpha and Beta angles. Each coil on the stator was separated into the up and down operation range. The rotor position was controlled by the current, which consisted of the variable Alpha and Beta angles.

In Fig. 2, the range of the tilt was decided by the angle between the centers of the up and down coils. Moreover, the opening diameter of the stator was calculated as angle up to no effect of the cogging torque between the stator opening part and the magnet for the rotor tilt.

\section{Torque Analysis}

The torque characteristics of the Multi-D.O.F. SPM were analyzed using the position holding torque and the rotating torque.

In Fig. 4, the holding torque must be stable in order to 


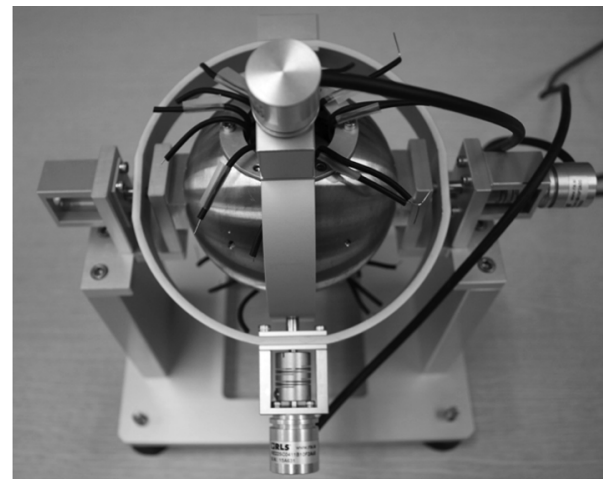

(a)

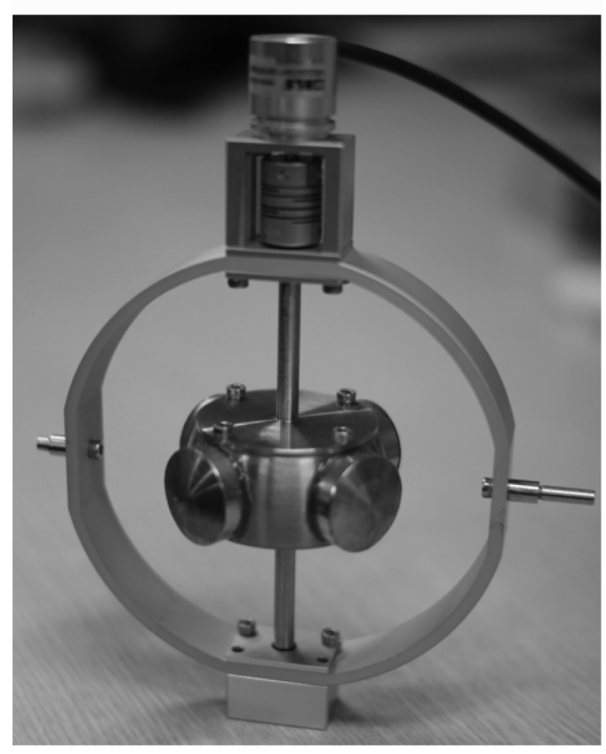

(b)

Fig. 1. Multi-degrees of the Freedom Surface Permanent-magnet Motor. (a) assembled motor (b) spherical shaped rotor.

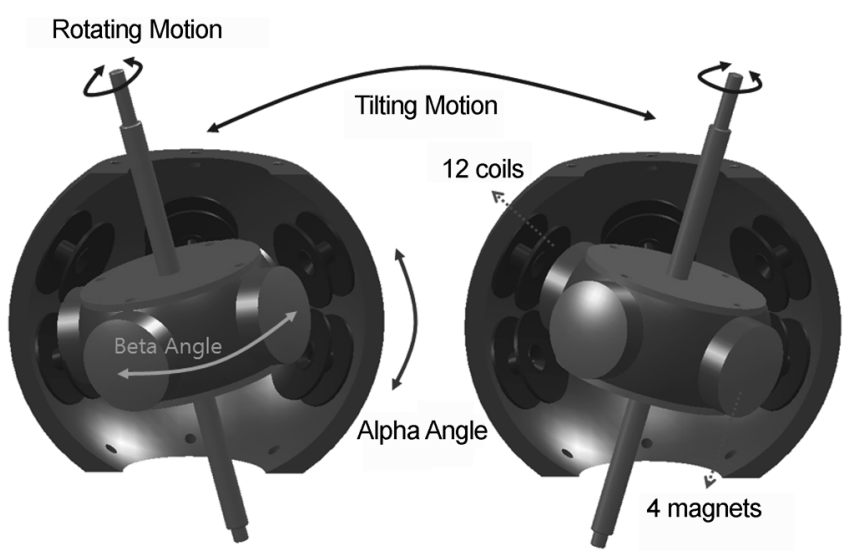

Fig. 2. Operation Characteristics.

precisely and immovably position the rotor. In a previous study, the characteristics of this holding torque were identified using the angle between coils. These characteristic were improved by using the following modified current

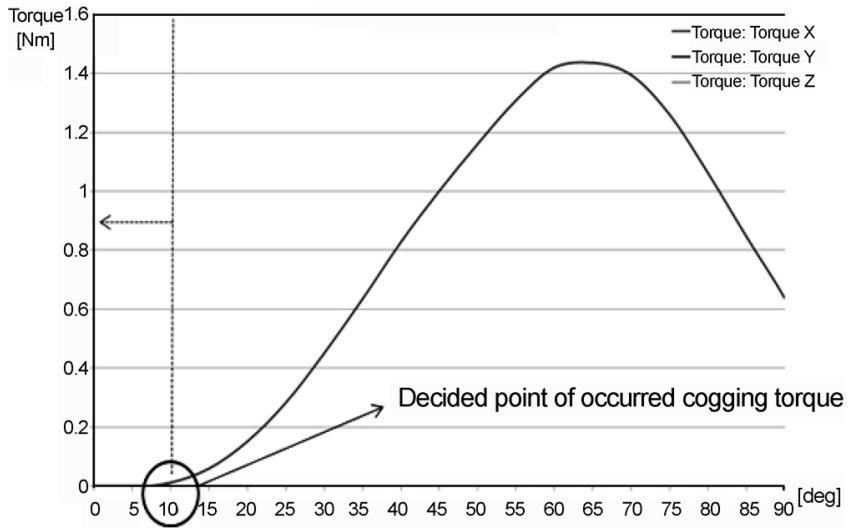

Fig. 3. Analysis of the cogging torque in order to determine the diameter of the stator opening.

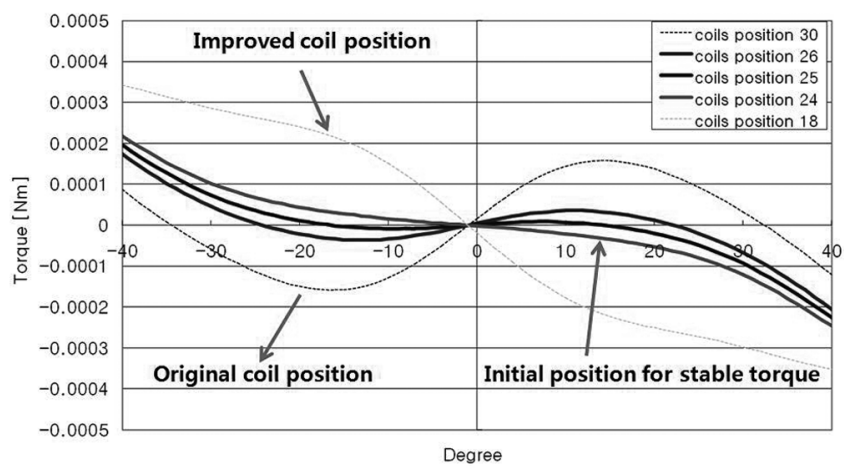

Fig. 4. Holding torque characteristics.

function: [5].

$$
\begin{aligned}
l_{\kappa \nu}= & I_{\mu} \cos \frac{\pi}{4}+(v) \frac{3}{2} \Gamma \times \cos \left(\beta-\frac{\pi}{3}(\kappa-1)\right) \\
& \times 2(\omega \tau+\beta)-\frac{2}{3} \pi(\kappa-1)
\end{aligned}
$$

$\Gamma:$ inverse function, compensated command alpha $\sigma$ : coefficients matrix

$n$ : order of polynomial

The analysis of the continuous operation characteristics was necessary because the Multi-D.O.F. SPM must continuously rotate while the rotor tilts at the command position. The torque profile and the torque ripple were analyzed using a simulation.

Unlike the 1-D.O.F. motor, which rotated on only one axis, the multi-D.O.F. SPM had different torque ripple profiles at the $\mathrm{X}, \mathrm{Y}$, and $\mathrm{Z}$ axis. Therefore, the torque profiles of each axis were analyzed. The torque characteristics of the z-axis were analyzed using various magnetization patterns for the permanent-magnet in the simulated model. Generally, the magnetization pattern of the permanent- 


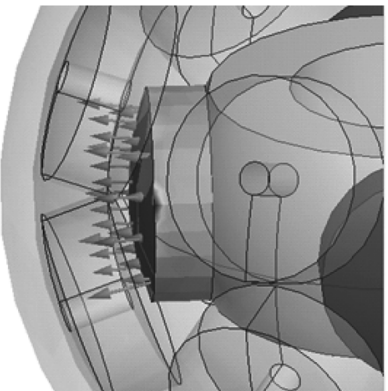

(a)

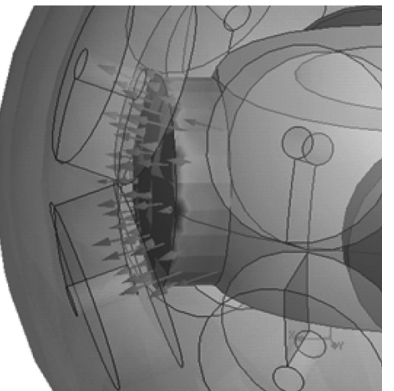

(b)
Fig. 5. Magnetization patterns using the simulation. (a) Parallel pattern (b) Spherical pattern.

magnet causes the distribution of the flux density in the air-gap and is an important factor in reducing the ripples in the pulsating torque $[6,7]$.

In this study, two types of magnetization patterns, parallel and spherical, were used for the simulation, in Fig. 5. The spherical magnetization pattern was a special pattern for the multi-D.O.F. SPM with a spherical rotor and stator.

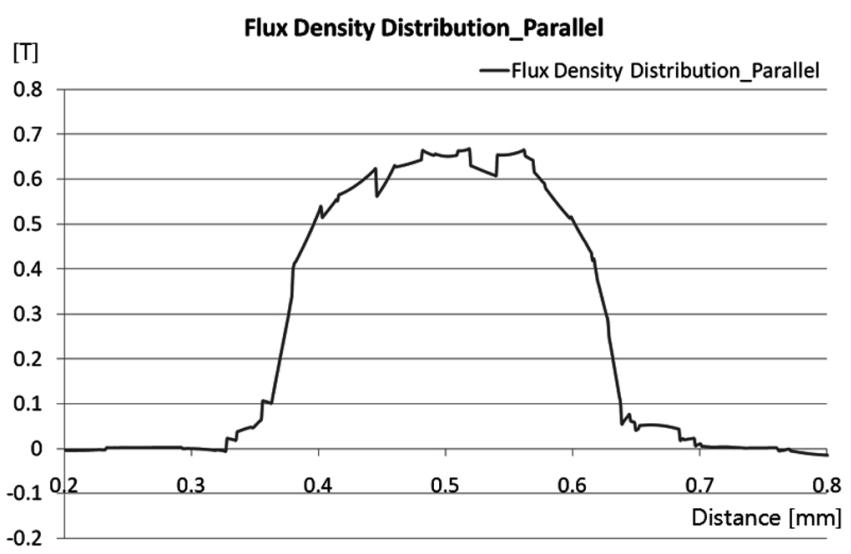

Fig. 6. Flux density distribution of the parallel magnetization pattern in the air-gap.

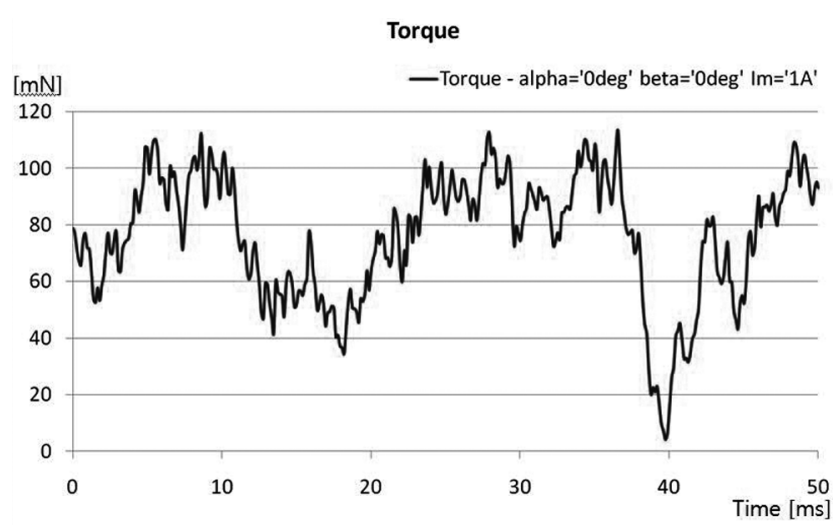

Fig. 7. Results of the torque simulation of the parallel magnetization pattern.
Each model simulated each rotor position at $\alpha=0$ deg., $\beta=0$ deg.

\subsection{Parallel Magnetization Pattern}

Fig. 6 shows the flux density distribution of the parallel magnetization pattern in the air-gap. Fig. 7 shows the torque and ripples analysis. The magnitude of the flux density had an rms value of $0.4122 \mathrm{~T}$ for the parallel magnetization pattern. The average torque was 76.1251 $\mathrm{mN}$, and the torque ripple was $77.878 \mathrm{mN}$ in terms of the peak to peak value.

\subsection{Spherical Magnetization Pattern}

Fig. 8 shows the flux density distribution of the parallel magnetization pattern in the air-gap. Fig. 9 shows the analysis of the torque and ripples. The magnitude of the flux density had an rms value of $0.3546 \mathrm{~T}$ for the parallel magnetization pattern. The average torque was 81.5815 $\mathrm{mN}$, and the torque ripple was $72.4383 \mathrm{mN}$ for the peak to peak value.

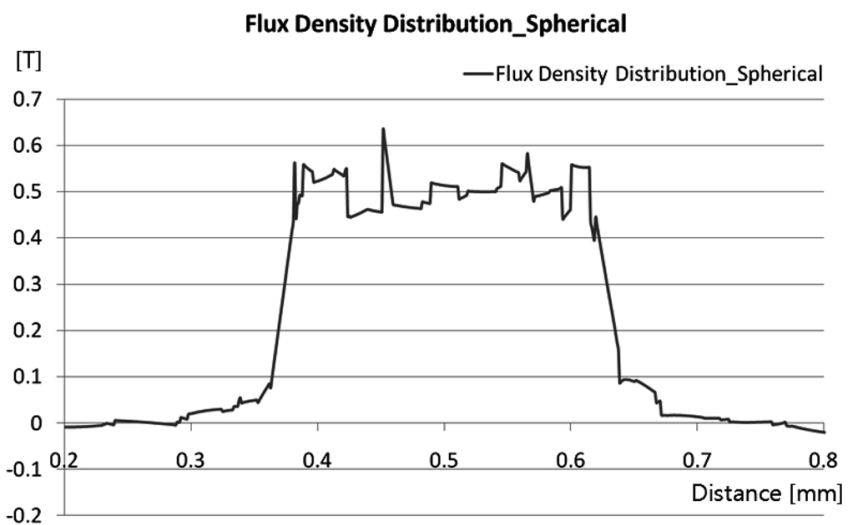

Fig. 8. Flux density distribution of the spherical magnetization pattern in the air-gap.

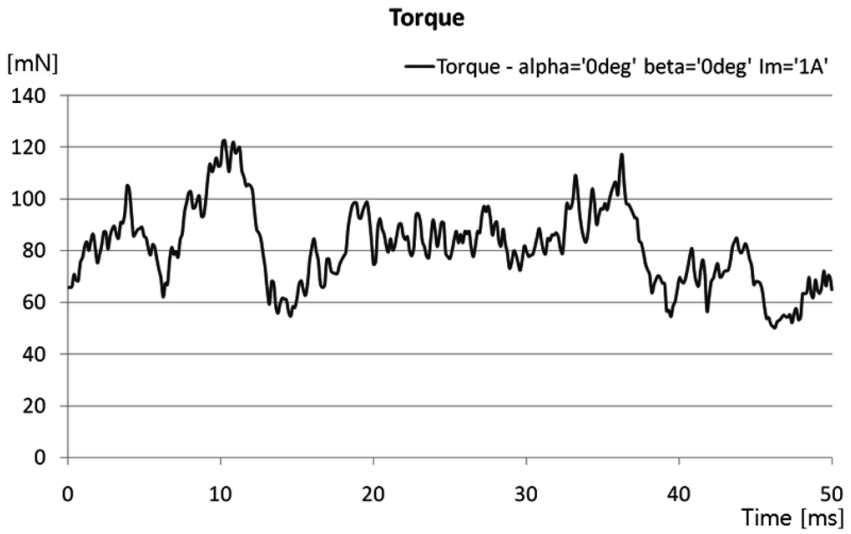

Fig. 9. Results of the torque simulation of the spherical magnetization pattern. 


\section{Discussion and Conclusion}

The magnetization pattern of the permanent-magnet affected the torque quality. The rms torque of the parallel magnetization pattern was smaller than the spherical magnetization pattern. Although the rms flux density of the parallel magnetization pattern was higher than the spherical magnetization pattern, the peak to peak value of the torque ripple was bigger than the spherical magnetization pattern. In this research, the torque characteristics affected the magnetization pattern of the Multi-D.O.F. SPM.

\section{Acknowledement}

This work was supported by the National Research Laboratory (NRL) through a grant provided by the Ministry of Education, Science \& Technology (MEST) in contract number R0A-2008-000-20048-0. Additionally, this work was partially supported by the Manpower Development
Program for Energy \& Resources supported by the Ministry of Knowledge and Economy (MKE), Republic of Korea.

\section{References}

[1] F. C. Williams, E. R. Laithwaite, and L. S. Piggott, Proc. Inst. Elec. Eng. (London), 104-A, 102 (1956).

[2] G. Vachtsevanos, K. Davey, and L. M. Lee, Control System Magazine 9 (1987).

[3] K. Kaneko, I. Yamada, and K. Ita, J. Dyn. Syst-t. ASME Measurement and Control 111, 398 (1989).

[4] K. M. Lee and C. K. Kwan, IEEE Robot. Autom. Mag. 7, 175 (1991).

[5] D. W. Kang, W. H. Kim, S. C. Go, C. S. Jin, S. H. W, D. H. Koo, and J. Lee, IEEE Trans. Magn. 45, 2819 (2009).

[6] T. M. Jahns and W. L. Soong, IEEE T. Ind. Electron. 43, 321 (1996).

[7] S. H. Lee, H. R. Cha, and H. W. Cho, Journal of Electrical Engineering \& Technology 3, 379 (2008). 